\title{
Postoperative intussusception following transverse colostomy: A case report with its pathogenesis
}

\author{
Shakya VC', Agrawal CS ${ }^{2}$, Koirala $\mathrm{R}^{\mathbf{3}}$, Khaniya $\mathrm{S}^{1}$, Shrestha $\mathrm{AB}^{4}$, Adhikary $\mathrm{S}^{2}$
}

${ }^{1}$ Assistant Professor, ${ }^{2}$ Professor, ${ }^{3}$ Associate Professor, ${ }^{4}$ Senior Resident, Department of Surgery, B. P. Koirala Institute of Health Sciences, Dharan, Nepal

\begin{abstract}
Postoperative idiopathic intussusception is considered to be a distinct entity, and has been reported following different operations. We present a 45-year-old female with postoperative ileoileal intussusception following a transverse loop colostomy for sigmoid volvulus, in which there was a kinked loop of bowel forming the lead point. The pathogenesis and literature review of this disorder is discussed.
\end{abstract}

Key words: postoperative intussusception, lead point, kink, motility disorder

Tntussusception is rare in the adult patient, Irepresenting only $1 \%$ of all cases of small bowel obstruction and $5 \%$ of all cases of bowel obstruction ${ }^{1}$. In most cases, there is an underlying lesion as a lead point $^{2}$. Postoperative idiopathic intussusception is now considered to be a distinct entity ${ }^{3}$. Here we present a case of an idiopathic ileoileal intussusception following an emergency transverse loop colostomy for sigmoid volvulus. Postoperative intussusception has never been reported following a colostomy, and with this report, the pathogenesis is discussed along with a review of literature.

\section{Case report}

A 45-year-old female presented to the emergency of B. P. Koirala Institute of Health Sciences with history of not passing stool and flatus for 2 days, gradually progressive abdominal distension and bilious vomiting. Her plain radiograph of the abdomen showed a typical pneumatic tyre appearance. She was subjected to an exploratory laparotomy which confirmed sigmoid volvulus, the sigmoid was resected, and a descending colo-rectal anastomosis was done, along with a protective transverse loop colostomy. Her initial postoperative course was unremarkable, the stoma started to function; the gastric aspirate was minimal in amount. However, from the $4^{\text {th }}$ postoperative day, the stoma output decreased drastically, she had progressive abdominal distension. Distension later subsided slowly, but the gastric aspirate increased suddenly even up to 2 liters per day. She also had hypokalemia on the $6^{\text {th }}$ postoperative day $(\mathrm{mEq} / \mathrm{L})$ which was gradually corrected. However, the gastric aspirate continued to be in higher amounts and, there was no stoma output. An $\mathrm{x}$-ray of the abdomen was done, which showed multiple air-fluid levels. Without undue delay, she was subjected to a re-exploration on the $9^{\text {th }}$ postoperative day. There was an ileoileal intussusception, about 2 feet above the ileocecal junction with grossly dilated proximal bowel loop, and collapsed distal bowel (Fig 1). The intussusception was reduced gradually, and there was a loop of bowel adhered to each other which formed a kink, and hence a lead point (Fig 2). Adhesiolysis was done. There was no palpable intraluminal lesion, the bowel was healthy, and after decompression of the bowel, the abdomen was closed. Postoperatively, the stoma started to function well, the gastric aspirate decreased. On the $3^{\text {rd }}$ day of re-exploration, the Ryle's tube was taken out, and she was orally allowed. She gradually recovered well; she was discharged on the $15^{\text {th }}$ postoperative day of the initial operation.

Correspondence

Vikal Chandra Shakya

Department of Surgery

B. P. Koirala Institute of Health Sciences, Dharan, Nepal

E-mail: vikalcsh@yahoo.com 


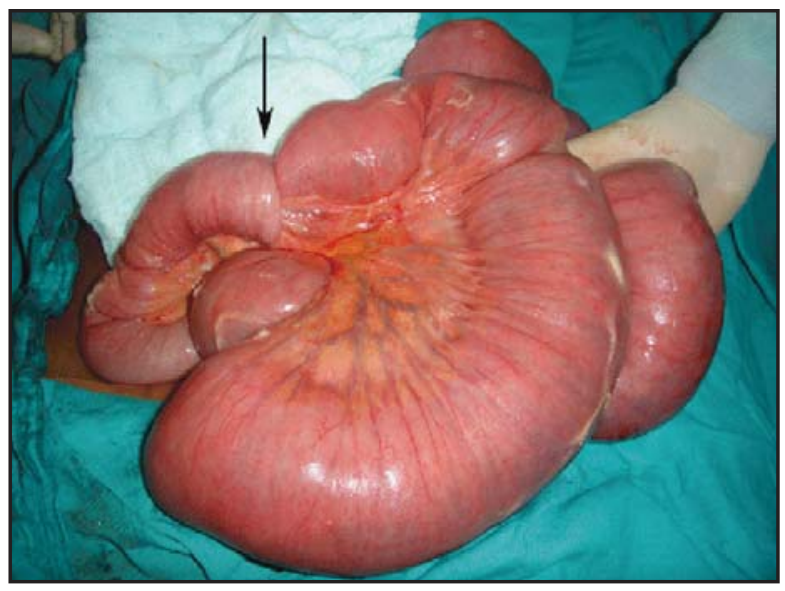

Fig 1: Laparotomy showing the ileoileal intussusception (arrow).

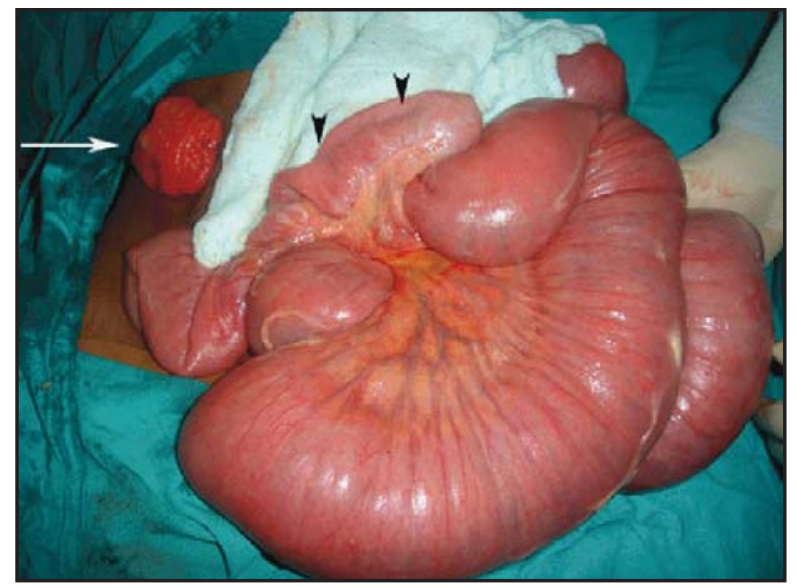

Fig 2: On reducing the intussusception, a loop of bowel (arrowheads) was found to be adhered to each other forming a kink, which formed the lead point causing the intussusception. The transverse colostomy (white arrow) is seen.

\begin{abstract}
Discussion
Postoperative idiopathic intussusception is rare in the adults $^{2,3}$. Our literature search has found out 56 articles on adult postoperative intussusceptions till date, most of which are isolated case reports. It has been reported after various operations ${ }^{4-13}$. The most frequent one is following gastrojejunostomy (either alone or with isoperistaltic loop or Roux-en-Y reconstruction for a Billroth II gastrectomy) as a clinical presentation of jejunogastric intussusception and after jejunoileal bypass for morbid obesity ${ }^{4,5}$. Other conditions included suture lines following trauma, previous jejunostomy sites, long intestinal tubes, after pancreaticoduodenectomy, hysterectomy, aortic aneurysm repair, closure of duodenal ulcer perforation and truncal vagotomy ${ }^{6-}$ 13. Postoperative intussusception has been reported in infants following enterostomy but this entity has never been reported in an adult ${ }^{14}$.
\end{abstract}

Postoperative idiopathic intussusception in the adult appears to be a different surgical entity compared to conventional adult intussusception presenting de novo; it typically occurs in adults 45 to 51 years old, occurs most commonly in the jejunojejunal region and develops 4 to 5 days postoperatively ${ }^{15}$. It may be related to a variety of factors as intrabdominal adhesions, submucosal oedema, presence of suture lines, electrolyte imbalance, chronic dilatation of the intestine or abnormalities in motor activity during the postoperative period ${ }^{3,15,16}$. Reymond et al postulated two different mechanisms of intussusception: a functionally noncontractile part of the intestinal wall may develop as an indurated area or a region of diameter change in the bowel, or a mechanical linkage of two nonadjacent bowel segments may form an intraluminal or extraluminal lead point in the postoperative period ${ }^{17,18}$. The second setting may be caused by any extraluminal focal adhesions between bowel loops. These effects lead to a kinking of the bowel which would produce intussusception with continued normal peristalsis/hyperperistalsis. Postoperative motility disorder may be another cause, especially following vagotomy ${ }^{13,16}$. In our patient the development of adhesions between two limbs of a bowel loop served as a stimulating factor for producing the initial kink and the lead point. It might have been reinforced by some motility disorder causing discoordinate contractions and a massive discrepancy in the lumen of the proximal and distal bowel, thus producing antegrade invagination.

The diagnosis of postoperative intussusception can be difficult, and a high index of suspicion is necessary for its early detection. The classic clinical triad of conventional intussusception consisting of abdominal pain, palpable sausage shaped mass, and currant-jelly stools is rarely present in the postoperative patient; the abdominal pain is usually attributed to the surgical wound, and the most important symptoms are vomiting and abdominal distension. So there lies a high likelihood of potential mishaps. Awareness of this disorder is the most important factor making the correct and prompt diagnosis and avoidance of the potentially high morbidity and mortality. Although ultrasonography, computed tomography and barium contrast have been found to be helpful in the diagnosis of postoperative intussusception, prompt surgical exploration remains the best diagnostic and therapeutic approach ${ }^{6,13,16}$.

Treatment of postoperative small bowel intussusception is surgical, either via laparoscopy or laparotomy. It 
usually requires reduction of the intussusception and lysis of all accompanying adhesions $\mathrm{s}^{3,13,15,16}$. If reduction is not possible or there is evidence of vascular compromise, the involved bowel may need to be resected. Contrary to other conventional forms of intussusception, in adults resection is not mandatory and recurrence has not been documented ${ }^{13,15,16}$.

\section{Conclusion}

Postoperative intussusception is rare in the adult. This report presents a case of postoperative intussusception developing after a transverse colostomy. It occurred probably due to postoperative adhesions resulting in a kink between two bowel loops. Diagnosis of postoperative intussusception is difficult and it is best treated by prompt surgical intervention. Reduction of intussusception and lysis of adhesions is usually enough.

\section{References}

1. Ercan N, Haciyanli M, Yildirim M, Sayhan H, Vardar E, Polat AF. Intussusception in adults: an unusual condition and challenging condition for surgeons. Int J Colorectal Dis. 2005;20(5):4526.

2. Barussaud M, Regenet N, Briennon X, et al. Clinical spectrum and surgical approach of adult intussusceptions: a multicentric study. Int J Colorectal Dis. 2006;21(8):834-9.

3. Sarr MG, Nagorney DM, Mcllrath DC. Postoperative intussusception in the adult: a previously unrecognized entity? Arch Surg. 1981;116(2):144-8.

4. Loizou MC, Koundourakis SS, Kollias VD, Panayotides HC, Papagrigoriadis SS. Jejunojejunal intussusception after Roux-en-Y gastrojejunostomy: a rare cause of postoperative bowel obstruction in an adult: case report. Eur J Surg. 1994;160(8):451-2.

5. Edwards MA, Grinbaum R, Ellsmere J, Jones $\mathrm{DB}$, Schneider BE. Intussusception after Rouxen-Y gastric bypass for morbid obesity: case report and literature review of rare complication. Surg Obes Relat Dis. 2006;2(4):483-9.
6. Chong TJ, Victorino GP. CT diagnosis of postoperative intussusception after penetrating abdominal trauma. J Gastrointest Surg. 2004;8(4):387-8.

7. Wu TH, Lin $\mathrm{CW}$, Yin WY. Jejunojejunal intussusception following jejunostomy. J Formos Med Assoc. 2006;105(4):355-8.

8. Brown EO, Miller JM. Retrograde jejunal intussusception following the use of a Cantor intestinal tube. Am J Proctol Gastroenterol Colon Rectal Surg. 1981;32(6):28-33.

9. Sedgwick CE, Vernon JK. Jejunojejunal intussusception: an unusual complication after pancreatodudoenectomy. Am J Surg. 1970;119(6):752-3.

10. Reddy NS, Rajaram P. Intussusception: a rare complicationfollowingabdominalhysterectomy. Int J Gynaecol Obstet. 1989;30(3):279-81.

11. Sowemimo OA. Multiple intussusceptions after aortic aneurysm repair. J Am Coll Surg. 2006;202(5):844-5.

12. Maroju NK, Srinivasan K, Kadambari D, Vaithiswaran V. Postoperative intussusception in the adult: a case report. Trop Gastroenterol. 2004;25(1):40-1.

13. Zbar AP, Murphy F, Krishna SM. Adult postoperative intussusception: a rare cause of small bowel obstruction. South Med J. 2007;100(10):1042-4.

14. Hsieh TK, Chen AC, Wu SF, Chen W. Postoperative intussusception in children with enterostomy. Acta Paediatr Taiwan. 2005;46(3):166-9.

15. Agha FP. Intussusception in adults. AJR Am J Roentgenol. 1986;146(3):527-31.

16. Eke N, Adotey JM. Postoperative intussusception, causal or casual relationships? Int Surg. 2000;85(4):303-8.

17. Reymond RD. The mechanism of intussusception: a theoretical analysis of the phenomenon. Br J Radiol. 1972;45(529):1-7.

18. Reymond RD. A mechanism of the kink formation which precedes intussusception. Invest Radiol. 1971;6(1):61-4. 